\title{
Desflurane differentially affects the release of proinflammatory cytokines in plasma and bronchoalveolar fluid of endotoxemic rats
}

\author{
KIM A. BOOST ${ }^{1}$, CHRISTIAN HOFSTETTER ${ }^{1}$, MICHAEL FLONDOR ${ }^{1}$, CHRISTIAN BETZ $^{1}$, \\ MARKUS HOMANN $^{1}$, JOSEF PFEILSCHIFTER ${ }^{2}$, HEIKO MUEHL ${ }^{2}$ and BERNHARD ZWISSLER ${ }^{1}$ \\ ${ }^{1}$ Department of Anaesthesiology, Intensive Care and Pain Therapy and ${ }^{2}$ Pharmazentrum Frankfurt/ZAFES, \\ Johann Wolfgang Goethe-University, 60590 Frankfurt am Main, Germany
}

Received December 8, 2005; Accepted January 30, 2006

\begin{abstract}
Previous studies have indicated that volatile anaesthetics can attenuate the inflammatory response to lipopolysaccharide (LPS) and other proinflammatory stimuli in vitro and in vivo. Thus far, no studies are available on the influences of desflurane on the cytokine-release. We therefore aimed to investigate the effects of desflurane on the systemic and pulmonary release of proinflammatory cytokines in endotoxemic rats. Eighteen anaesthetized and ventilated SpragueDawley rats were randomly assigned to the following groups: LPS-only: Six animals received LPS (5 mg/kg, i.v.) with no further intervention. LPS-Desflurane: Six animals received continuous inhalation of 1MAC Desflurane before and during endotoxemia with LPS (5 mg/kg, i.v.). Sham: Six animals served as control without inhalation of desflurane and endotoxemia. After $4 \mathrm{~h}$, levels of tumor necrosis factor- $\alpha$ (TNF- $\alpha$ ), interleukin-1ß (IL-1ß) and interleukin-6 (IL-6) in plasma and bronchoalveolar fluid were analyzed. Nitrite production as a readout for nitric oxide (NO) release from alveolar macrophages was measured by Griess assay. IкB- $\alpha$ degradation and iNOS-protein in macrophage homogenates were determined by Western Blotting. Inhalation of desflurane during endotoxemia showed a significant decrease in release of the proinflammatory cytokines TNF- $\alpha(-61 \%, \mathrm{P} \leq 0.05)$ and IL- $1 \beta$ $(-47 \%, \mathrm{P} \leq 0.05)$ in plasma as compared to LPS-only group, whereas the release of IL- 6 was not significantly affected by desflurane. Within the lung, the NO-release was notably increased in supernatants of cultured alveolar macrophages from desflurane-group compared to both LPS-only and Sham group. IкB- $\alpha$ degradation in alveolar macrophages was
\end{abstract}

Correspondence to: Dr Kim Alexander Boost, Department of Anaesthesiology, Intensive Care and Pain Therapy, Klinikum der Johann Wolfgang Goethe-Universität, Frankfurt am Main, TheodorStern-Kai 7, 60590 Frankfurt am Main, Germany

E-mail: k.boost@med.uni-frankfurt.de

Key words: desflurane, rat, lipopolysaccharide, interleukin-1ß, tumor necrosis factor- $\alpha$ impaired in the Desflurane-group as compared to the LPS-only group. Our data implicate that inhalation of 1MAC Desflurane during experimental endotoxemia differentially affects the inflammatory response in rats.

\section{Introduction}

Inflammation caused by endotoxemia or traumatic injury leads to a strong release of proinflammatory cytokines in bronchoalveolar fluid and plasma $(1,2)$. Several previous studies have indicated that exposure to volatile anaesthetics, such as isoflurane, enflurane and halothane, attenuates the inflammatory response through decreasing the cytokine release from alveolar macrophages and monocytes following stimulation with lipopolysaccharide (LPS) (3-5). Moreover, volatile anaesthetics modulate the immune response by decreasing neutrophil functions in vitro (6) and cytokine release by peripheral mononuclear cells (7). More recently, we described that even a brief inhalation of isoflurane $(50 \mathrm{sec})$ markedly decreased the levels of proinflammatory cytokines in plasma of endotoxemic rats (8).

Among the inhalational anaesthetics little is known about the influence of desflurane on the inflammatory response of alveolar macrophages and mononuclear cells. To our knowledge, there are no studies concerning the impact of desflurane on the cytokine release during inflammation. Because of the growing use of desflurane in clinical practice, the evaluation of possible immunomodulative properties is of sustained interest for patients with systemic inflammatory response syndrome (SIRS) or acute lung injury/ARDS. Therefore, the aim of this study was to examine the effects of desflurane on the inflammatory response indicated by the release of proinflammatory cytokines in plasma and bronchoalveolar fluid during experimental endotoxemia with LPS.

\section{Materials and methods}

Animals and anaesthesia. All animal experiments were approved by the governmental board for the care of animal subjects (Regierungspräsidium Darmstadt, Germany) in accordance to the Declaration of Helsinki and received care in compliance with the 'Guide for the Care and Use of 
Table I. Heart rate, mean arterial blood pressure and BALF cell count.

\begin{tabular}{|c|c|c|c|c|c|}
\hline Time & Baseline & $1 \mathrm{~h}$ & $2 \mathrm{~h}$ & $3 \mathrm{~h}$ & $4 \mathrm{~h}$ \\
\hline \multicolumn{6}{|l|}{$\operatorname{HR}[1 / \mathrm{min}]$} \\
\hline Sham & $338(26)$ & $333(27)$ & $333(34)$ & $327(18)$ & $320(18)$ \\
\hline LPS-only & $356(44)$ & $348(55)$ & $354(50)$ & $364(24)$ & $372(43)$ \\
\hline Desflurane & $352(39)$ & $330(39)$ & $344(29)$ & $364(23)$ & $362(31)$ \\
\hline \multicolumn{6}{|l|}{ MAP $[\mathrm{mmHg}]$} \\
\hline Sham & $115(35)$ & $105(14)$ & $97(23)$ & $97(23)$ & $96(24)$ \\
\hline LPS-only & $103(28)$ & $91(28)$ & 89 (29) & $90(22)$ & $98(7)$ \\
\hline Desflurane & $129(30)$ & $101(26)$ & $91(24)$ & $109(20)$ & $109(13)$ \\
\hline \multicolumn{6}{|c|}{ TCC-BALF $\left[\times 10^{6} / \mathrm{ml}\right]$} \\
\hline Sham & & & & & $3.8(0.9)$ \\
\hline LPS-only & & & & & $2.8(0.4)^{\mathrm{a}}$ \\
\hline Desflurane & & & & & $1.9(0.2)^{\mathrm{b}}$ \\
\hline
\end{tabular}

TCC-BALF $=$ total cell count - bronchoalveolar lavage fluid. Each value represents mean $( \pm$ SD), Kruskal-Wallis one way analysis of variance

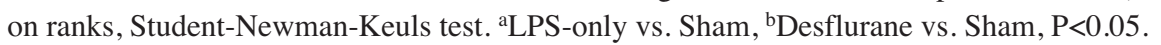

Laboratory Animals', National Academic Press, Washington D.C. 1996. Male Sprague-Dawley rats $(n=18)$ were obtained from Harlan Winkelmann (Borchen, Germany; mean body weight \pm SEM, $490 \pm 30 \mathrm{~g}$ ) and kept on a 12-h light/dark cycle with free access to food and water. Rats were anaesthetized initially by i.p. injection of pentobarbital (Narcoren, Merial, Halbergmoos, Germany; $50 \mathrm{mg} / \mathrm{kg}$ ) plus fentanyl (JanssenCilag, Neuss, Germany; $0.05 \mathrm{mg} / \mathrm{kg}$ ). Unconscious rats were tested for sufficient depth of anaesthesia by tail clamping, weighed and then placed supine on a heating pad. A tracheotomy was immediately performed and a $13 \mathrm{G}$ cannula (ID: $2.0 \mathrm{~mm}$, OD: $2.5 \mathrm{~mm}$, Abbott, Wiesbaden, Germany), modified with a standard connector for pediatric endotracheal tubes, was endotracheally inserted. Subsequently, rats were ventilated with an infant ventilator (Stephanie ${ }^{\circledR}$, Stephan, Gackenbach, Germany) using pressure controlled ventilation: $\mathrm{p}_{\max } 1.6 \mathrm{kPa}$, PEEP $0.4 \mathrm{kPa}$, respiratory rate $40 / \mathrm{min}$, inspiratory oxygen fraction $0.24, \mathrm{~T}_{\mathrm{I} / \mathrm{E}}: 1: 2$. Respiratory settings were adjusted to maintain normocapnia according to hourly performed arterial blood gas analyses. The ventilator was modified for administration of volatile anaesthetics with a standard vapor (Draeger Medical, Lübeck, Germany). A temperature probe was inserted rectally in order to keep body temperature constant at $37-38^{\circ} \mathrm{C}$ throughout the experiment. Fluid- filled polyurethane catheters (ID $0.58 \mathrm{~mm}$, OD $0.96 \mathrm{~mm}$, SIMS Portex Ltd., Hythe, UK) were inserted in the right femoral vein and artery for infusion of anaesthetics and withdrawal of blood samples, respectively.

Experimental design. Surgical preparation was followed by a stabilization period of $15 \mathrm{~min}$. Thereafter, animals were randomly assigned to the study-groups. In the LPS-only and Sham-group anaesthesia was maintained by continuous i.v. infusion of pentobarbital $(5-10 \mathrm{mg} / \mathrm{kg} / \mathrm{h})$ and fentanyl $(2.5-5 \mu \mathrm{g} / \mathrm{kg} / \mathrm{h})$ and in the Desflurane-group by desflurane (Baxter, Unterschleissheim, Germany; 1.0 minimum alveolar concentration (MAC) (6\%) and fentanyl (2.5-5 $\mu \mathrm{g} / \mathrm{kg} / \mathrm{h}$, i.v.). Concentration of desflurane was measured continuously by using a monitor for volatile anaesthetics (Vamos, Draeger Medical, Lübeck, Germany). Thereafter, all animals except the Sham-group received i.v. endotoxin (lipopolysaccharide, LPS, E. coli (055:B5), Sigma, Deisenhofen, Germany, $5 \mathrm{mg} / \mathrm{kg}$ ). After $4 \mathrm{~h}$ of endotoxemia, animals were exsanguinated and plasma samples were obtained.

Bronchoalveolar lavage (BAL). After thoracotomy, a bronchoalveolar lavage (BAL) was performed with 10 aliquots of $10 \mathrm{ml}$ sterile, nonpyrogenic phosphate-buffered saline (PBS; Serva, Heidelberg, Germany). Every rinse with a $10 \mathrm{ml}$ aliquot was performed standardized in $30 \mathrm{sec}$. The first aliquot of BALfluid samples was separated from the following aliquots and centrifuged. The supernatant was stored at $-80^{\circ} \mathrm{C}$ for further analysis. The remaining 9 BAL-fluid samples were pooled and centrifuged at $1500 \mathrm{rpm}$ for $10 \mathrm{~min}$, the resulting cellpellet resuspended and washed twice with PBS. The cells were then seeded in polystyrene plates for culture.

Plasma samples, nitrite assay, ELISA assay. The pooled blood samples were centrifuged at $5000 \mathrm{rpm}$ for $5 \mathrm{~min}$. Plasma was collected and stored at $-20^{\circ} \mathrm{C}$ till further analysis was performed.

BALF-derived cells were then suspended in RPMI-1640 supplemented with $100 \mathrm{U} / \mathrm{ml}$ penicillin, $100 \mu \mathrm{g} / \mathrm{ml}$ streptomycin, and 10\% FCS (Gibco-BRL, Eggenstein, Germany). Cells were seeded on a 24 -well polystyrene plate at $0.2 \times 10^{6}$ cells/well. After initial cultivation for $2 \mathrm{~h}$, non-adherent cells were removed. Adherent cells were regarded as alveolar macrophages and incubated with $0.5 \mathrm{ml}$ of the aforementioned medium. All incubations were performed at $37^{\circ} \mathrm{C}, 5 \% \mathrm{CO}_{2}$ in air.

After $24 \mathrm{~h}$ of incubation, cell culture supernatants were collected, centrifuged and the nitrite release by alveolar 
A

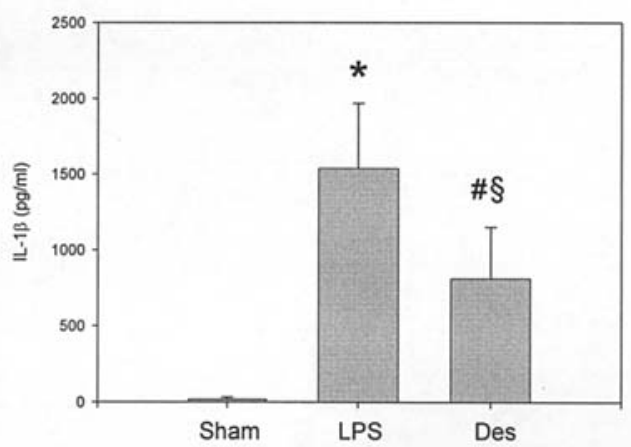

C

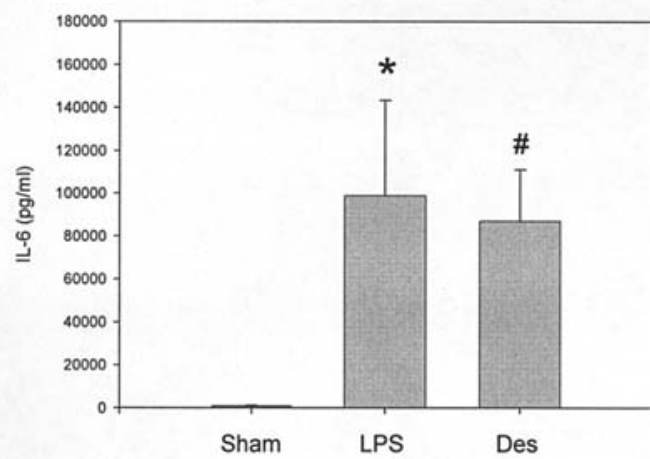

macrophages was analyzed as a read-out for inducible nitric oxide synthase (iNOS) activity in these cells. For this purpose nitrite concentrations in cell culture supernatants were determined by the Griess reaction. Briefly, $50 \mu 1$ of culture supernatants were mixed with $50 \mu 1$ of Griess reagent (Merck, Darmstadt, Germany). Absorbance at 540/595 nm and comparison with a nitrite standard gave nitrite concentrations in culture supernatants.

Plasma levels of rat TNF- $\alpha$, IL- 6 and IL- $1 \beta$ were determined by ELISA (R\&D-Systems, Wiesbaden, Germany) according to the manufacturer's instructions.

Western blot analysis. Briefly, cell homogenates from BALsample-derived alveolar macrophages were lysed on ice in lysis buffer (100 mM NaCl, $20 \mathrm{mM}$ TriCl, pH 7.8, 0.1\% NP-40) supplemented with a protease inhibitor cocktail (Roche Molecular Biochemicals). Homogenates were than centrifuged at $10000 \mathrm{rpm}, 10 \mathrm{~min}$ at $4^{\circ} \mathrm{C}$. Supernatants were collected and stored at $-80^{\circ} \mathrm{C}$ until further analysis. Total protein $(40 \mu \mathrm{g})$ was loaded per lane and separated in a $10 \%$ SDS-PAGE gel for I $\mathrm{KB}-\alpha$ and $7.5 \%$ SDS-PAGE gel for iNOS by standard electrophoresis at $100 \mathrm{~V}$. Proteins were then electrophoretically transferred to a nitrocellulose membrane. The membrane was subsequently blocked in $10 \%$ non-fat dry milk for $1 \mathrm{~h}$ at room temperature and incubated overnight at $4^{\circ} \mathrm{C}$ with primary antibodies to IkB- $\alpha$ (Santa Cruz Biotechnology) and iNOS (immunoblotting was performed as described, using a polyclonal anti-iNOS antibody (21) at a
B

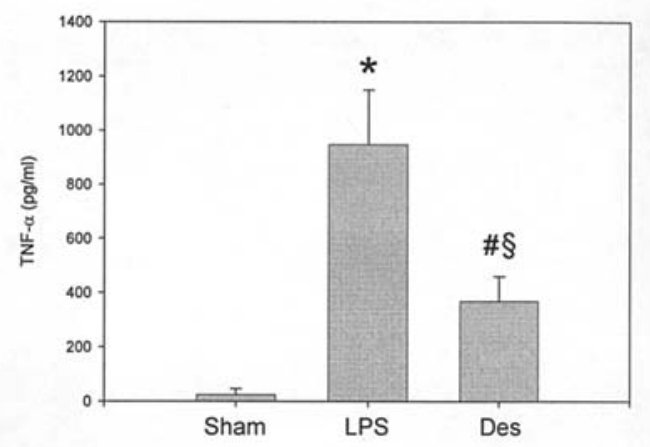

Figure 1. (A-C) Cytokine levels of TNF- $\alpha$, IL-1ß and IL-6 in plasma after $4 \mathrm{~h}$ of endotoxemia, determined by ELISA. Sham denotes the Sham-group consisting of rats anaesthetized with pentobarbital plus fentanyl and no further intervention. LPS denotes the LPS-only group consisting of rats anaesthetized with pentobarbital plus fentanyl during $4 \mathrm{~h}$ of endotoxemia with LPS $(5 \mathrm{mg} / \mathrm{kg}$ ). Des denotes the Desflurane-group consisting of rats exposed to Desflurane inhalation during $4 \mathrm{~h}$ of endotoxemia with LPS $(5 \mathrm{mg} / \mathrm{kg})$. Data are expressed as mean cytokine concentrations $\pm \mathrm{SEM}$; Comparison of all 3 groups (Sham, LPS-only and Desflurane, respectively). *, LPS-only vs. Sham; §, Desflurane vs. LPS-only; \#, Desflurane vs. Sham; $\mathrm{P}<0.05, \mathrm{n}=6$.

dilution of 1:1000 and 1:2000, respectively. After washing, horseradish peroxidase-conjugated secondary antibody was added at a dilution of 1:10000 and incubated at room temperature for $1 \mathrm{~h}$. After repeated washing, blots were subsequently developed using an enhanced chemiluminescence detection kit (Amersham) and exposed on autoradiographic film.

Statistics. Data are expressed as mean \pm SEM. Statistical analysis was performed with Sigma Stat (SPSS-Jandel 2.0 Scientific, San Jose, CA). Groups were compared with Kruskal-Wallis one way analysis of variance on ranks and the Student-Newman-Keuls test. Differences between groups were considered significant at $\mathrm{P} \leq 0.05$.

\section{Results}

Among all animals, there were no significant differences in mean arterial blood pressure (MAP), heart rate (HR) and ventilation values. Total cell count in bronchoalveolar lavage fluid (TCC-BALF) was significantly lower in the LPS-only and the Desflurane group compared to the Sham-group (Table I).

Release of cytokines in plasma and BAL. As compared to the Sham-group, $4 \mathrm{~h}$ of endotoxemia induced a significant increase of TNF- $\alpha$, IL- $1 \beta$ and IL- 6 in plasma and BAL samples of the LPS-only group (Fig. 1A-C). The administration of desflurane 
A

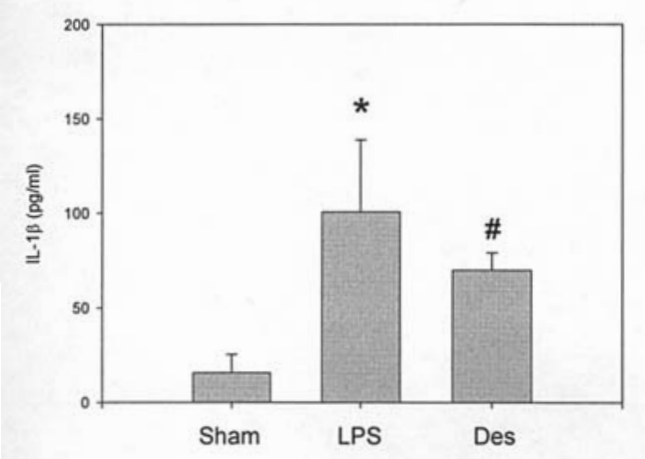

C

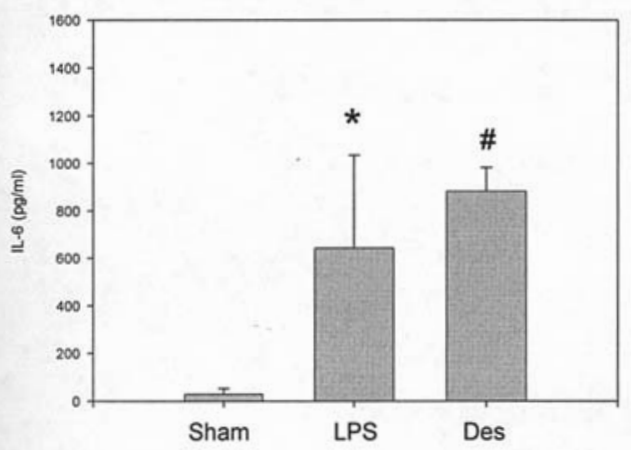

B

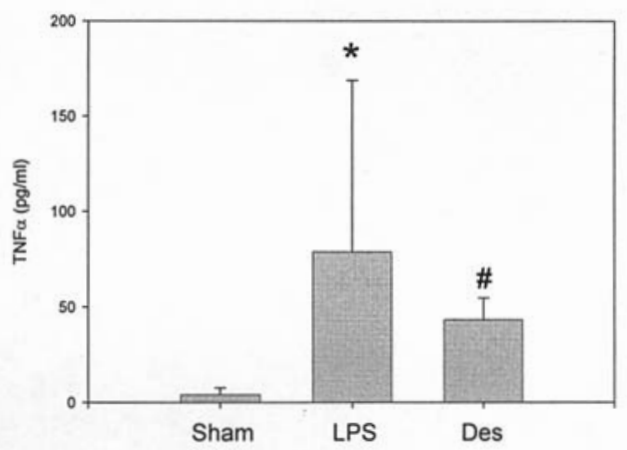

D

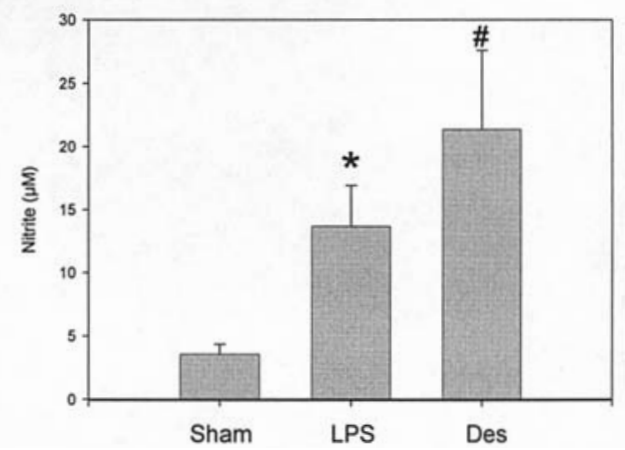

Figure 2. (A-C) Cytokine levels of TNF- $\alpha$, IL-1ß and IL-6 in BALF after 4 h of endotoxemia, determined by ELISA. Sham denotes the Sham-group consisting of rats anaesthetized with pentobarbital plus fentanyl and no further intervention. LPS denotes the LPS-only group consisting of rats anaesthetized with pentobarbital plus fentanyl during endotoxemia with LPS $(5 \mathrm{mg} / \mathrm{kg})$. Des denotes the Desflurane-group consisting of rats exposed to desflurane inhalation during endotoxemia with LPS $(5 \mathrm{mg} / \mathrm{kg}$ ). Data are expressed as mean cytokine concentrations \pm SEM; Comparison of all three groups (Sham, LPS-only and Desflurane, respectively). *, LPS-only vs. Sham; \#, Desflurane vs. Sham; $\mathrm{P}<0.05, \mathrm{n}=6$. (D) After 4 h of endotoxemia, alveolar macrophages were isolated and spontaneous production of nitrite, indicative of iNOS expression in these cells was analyzed by the Griess assay. Data are expressed as mean nitrite concentrations \pm SEM; *, LPS-only vs. Sham; \#, Desflurane vs. Sham; $\mathrm{P}<0.05, \mathrm{n}=6$.

during experimental endotoxemia significantly inhibited the release of TNF- $\alpha$ by $-61 \%(\mathrm{P} \leq 0.05)$ and IL- $1 \beta$ by $-47 \%$ $(\mathrm{P} \leq 0.05)$ in plasma (Desflurane-group) as compared to the LPS-only group and showed a strong trend in reducing the concentrations of both cytokines in the BAL. In contrast, desflurane did not affect IL-6 concentrations, either in plasma or in the BAL.

$N F-\kappa B$ activation in alveolar macrophages. We explored a possible mechanism for the effects of desflurane on the release of cytokines by examining its influence on the degradation of IкB- $\alpha$, the major inhibitor of NF-кB. As shown in Fig. 3B, the IкB- $\alpha$ level in macrophages was markedly reduced in the LPS-only group compared to the Sham-group, while in the Desflurane-group the IкB- $\alpha$ level was maintained and was similar to that of the Sham-group.

NO-production and iNOS-levels in alveolar macrophages. The production of NO from cultured alveolar macrophages was significantly increased in the LPS-only group compared to the Sham-group. Concurrently, there was a notable increase in NO-production in the Desflurane-group compared to the LPS-only group ( $\mathrm{p}=0.075$ ) (Fig. 2D). Accordingly, Western blotting of iNOS-protein in alveolar macrophages showed a highly increased level of cytosolic iNOS in the Desfluranegroup compared to LPS-only and Sham-group (Fig. 3A).

\section{Discussion}

Extensive release of proinflammatory cytokines in plasma and bronchoalveolar fluid are triggered by various inflammatory stimuli, such as endotoxin from gram negative bacteria, trauma or extracorporeal circulation during cardiosurgical intervention, respectively. In various patients this cytokine-release is frequently associated with the development of systemic inflammatory response syndrome (SIRS), sepsis or acute respiratory distress syndrome (ARDS) $(1,9-12)$. The severity of SIRS and ARDS is closely related to the extent of secreted proinflammatory cytokines $(11,13)$. Highly increased levels of proinflammatory cytokines, such as tumor necrosis factor- $\alpha$ (TNF- $\alpha$ ), interleukin-1ß (IL-1ß) and interleukin-6 (IL-6) can be found in plasma or bronchoalveolar lavage fluid (BALF) 
A

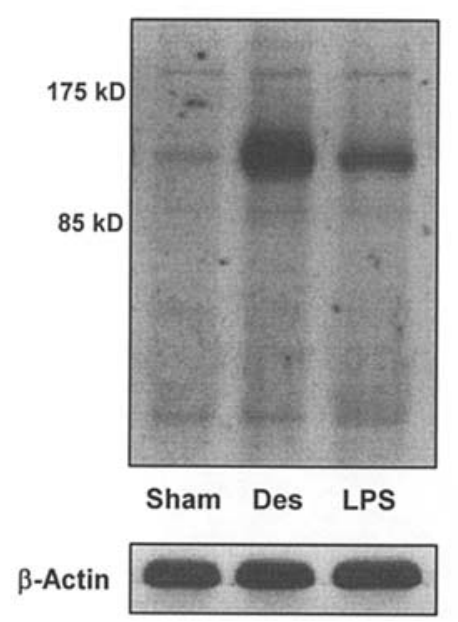

B

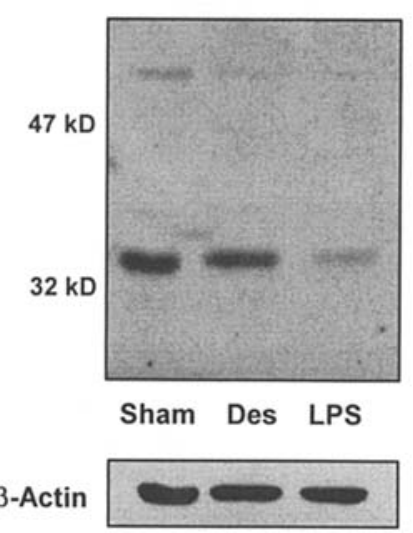

Figure 3. (A) Up-regulation of iNOS protein levels in alveolar macrophages determined by Western blot analysis. Sham denotes the Sham-group consisting of rats anaesthetized with pentobarbital plus fentanyl and no further intervention. LPS denotes the LPS-only group consisting of rats anaesthetized with pentobarbital plus fentanyl during endotoxemia with LPS $(5 \mathrm{mg} / \mathrm{kg})$. Des denotes the Desflurane-group consisting of rats exposed to desflurane inhalation during endotoxemia with LPS $(5 \mathrm{mg} / \mathrm{kg}$ ). Total cell lysates were immunoblotted with anti-iNOS antibodies. The data shown are representative for three

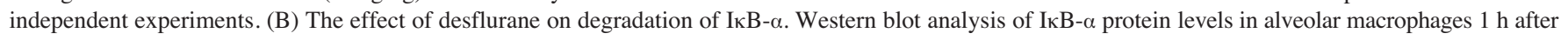
administration of LPS $(5 \mathrm{mg} / \mathrm{kg})$. The data shown are representative for two independent experiments.

during SIRS, sepsis or ARDS (12). Simultaneously, the released cytokines induce aggregation and influx of macrophages and neutrophils in the alveolar space to maintain and enhance the inflammation response $(12,14)$. IL-1ß plays a major role in the body's response to severe injury and endotoxin challenge and stimulates the secretion of both IL-6 and TNF- $\alpha$ (15).

This study demonstrates that inhalation of $1 \mathrm{MAC}$ Desflurane during $4 \mathrm{~h}$ of endotoxemia significantly inhibits the release of IL-1ß, and TNF- $\alpha$ in plasma compared to nonedesflurane-treated animals. The results are consistent with those of in vivo and in vitro studies, which examined the antiinflammatory effects of isoflurane, enflurane or halothane on the immune response during stimulation with LPS (3-6). Giraud and co-workers demonstrated that exposure to various volatile anaesthetics decreased the IL-1ß release from alveolar epithelial cells (16). Mitsuhata and co-workers showed that isoflurane inhibits the LPS-induced secretion of TNF- $\alpha$ and IL-1ß by peripheral mononuclear cells (7). As indicated in our pilot experiments, the peak of the proinflammatory cytokines TNF- $\alpha$ and IL- $1 \beta$ is located between 4 and $5 \mathrm{~h}$ after induction of experimental endotoxemia in rats with LPS (unpublished data). Based on these results, we chose the observation period of $4 \mathrm{~h}$ to exclude possible falsification by mounting or already declining cytokine levels of both mediators.

The BAL levels of IL-1ß and TNF- $\alpha$ during endotoxemia showed a consistent trend to be decreased by inhalation of desflurane. Although this effect failed to reach statistical significance, it strongly supports the view that inhalation of desflurane attenuates the inflammatory response during experimental endotoxemia by reducing the release of important proinflammatory cytokines both in plasma and BAL.

To further clarify the mechanisms which lead to the decreased cytokine secretion in plasma of endotoxemic rats treated by desflurane, we determined the activation of NF-кB in macrophages through the detection of IкB- $\alpha$. NF- $\kappa \mathrm{B}$ is the main transcription factor involved in the LPS-induced production of TNF- $\alpha$, IL- $1 \beta$ and IL- 6 and, therefore, represents a potential molecular target for the effects of volatile anaesthetics in macrophages and monocytes (17). Activation of $\mathrm{NF}-\kappa \mathrm{B}$ is mediated through degradation of his major inhibitor I $\mathrm{B}-\alpha$. Analysis by Western blotting revealed that a possible mechanism of the desflurane mediated effect is the reduced degradation of IкB- $\alpha$ in macrophages, followed by a decreased activation and translocation of NF- $\mathrm{KB}$. This corresponds with a previous study from De Rossi et al who showed a reduced activation of NF-кB during exposure to isoflurane (18). The finding in this study that desflurane did not affect the secretion of IL- 6 while significantly reducing the release of TNF- $\alpha$ and IL-1ß in plasma, can not be completely explained by the available data. It can be speculated, however, that this might be related to the incomplete inhibition of NF-кB shown by a reduced degradation of I $\kappa \mathrm{B}-\alpha$ or to other not measured pathways of IL-6 synthesis, independent of NF-кB. The degree of reduction seems to be adequate to significantly inhibit the release of TNF- $\alpha$ and IL-1 13 in plasma but not sufficient enough to attenuate the release of IL-6. We therefore assume that the lack of effect of desflurane on the release of IL-6 in plasma and BALF might be related to the incomplete inhibition of NF- $\mathrm{KB}$.

The release of NO showed a strong trend to be increased in alveolar macrophages in desflurane-treated animals in comparison to LPS-only group $(\mathrm{P}=0.075)$. This finding was corroborated by the fact that we identified an increase of iNOS-protein isolated from alveolar macrophages in desflurane-treated animals compared to LPS-only and Sham. The observed increase in iNOS-protein and NO-release in desflurane-treated animals is contradictory to several studies, which describe suppressive effects of volatile anaesthetics on 
the NO-release in vitro during stimulation with LPS (19). However, a possible explanation for this discrepancy has already been suggested (19). Incubation of J774 macrophages with a combination of LPS and IFN $\gamma$ in the presence of 1MAC Desflurane increased the NO-release of these cells. In contrast, incubation of the same cells with either LPS or IFN $\gamma$ in the presence of 1MAC Desflurane did not result in elevated NO-release. This shows in conclusion that the combination of both inflammatory stimuli modifies differentially the inflammatory response in contrast to their separate application. This partly explains the inconsistencies of in vivo and in vitro models and shows a marked advantage of in vivo models, which combine more components of the pathologic-physiologic inflammatory response. As shown in previous studies, the augmented release of NO decreases the secretion of IL-1ß and TNF- $\alpha$ from macrophages (20). Therefore, we hypothesize that the increased release of NO from alveolar macrophages might participate in the immune modulating effects of desflurane during inflammation.

In conclusion, our study suggests that desflurane differentially affects the release of proinflammatory cytokines in LPS-induced endotoxemia in rats, suggesting a modulative role in systemic inflammation. The underlying mechanism is presumably associated with a decreased degradation of I $\mathrm{KB}-\alpha$, followed by a reduced activation of NF-кB. Whether desflurane may also affect the inflammatory response in humans, requires further study.

\section{Acknowledgements}

The study was supported by a Grant of the August ScheidelStiftung, University of Frankfurt to C.H.

\section{References}

1. Bhatia M and Moochhala S: Role of inflammatory mediators in the pathophysiology of acute respiratory distress syndrome. J Pathol 202: 145-156, 2004.

2. Giannoudis PV: Current concepts of the inflammatory response after major trauma: an update. Injury 34: 397-404, 2003.

3. Plachinta RV, Hayes JK, Cerilli LA and Rich GF: Isoflurane pretreatment inhibits lipopolysaccharide-induced inflammation in rats. Anesthesiology 98: 89-95, 2003.

4. Helmy SA and Al-Attiyah RJ: The effect of halothane and isoflurane on plasma cytokine levels. Anaesthesia 55: 904-910, 2000.

5. Giraud O, Seince PF, Rolland C, Lecon-Malas V, Desmonts JM, Aubier M and Dehoux M: Halothane reduces the early lipopolysaccharide-induced lung inflammation in mechanically ventilated rats. Am J Respir Crit Care Med 162: 2278-2286, 2000 .
6. Moudgil GC, Allan RB, Russell RJ and Wilkinson PC: Inhibition, by anaesthetic agents, of human leucocyte locomotion towards chemical attractants. Br J Anaesth 49: 97-105, 1977.

7. Mitsuhata H, Shimizu R and Yokoyama MM: Suppressive effects of volatile anesthetics on cytokine release in human peripheral blood mononuclear cells. Int J Immunopharmacol 17: 529-534, 1995.

8. Hofstetter C, Flondor M, Boost KA, Koehler P, Pfeilschifter J, Zwissler B and Muehl H: A brief exposure to isoflurane (50 seconds) markedly impacts on plasma cytokine levels in endotoxemic rats. Int Immunopharm 5: 1519-1522, 2005.

9. Strunk V, Hahnenkamp K, Schneuing M, Fischer LG and Rich GF: Selective iNOS inhibition prevents hypotension in septic rats while preserving endothelium-dependent vasodilation. Anesth Analg 92: 681-687, 2001.

10. Taniguchi T, Shibata K and Yamamoto K: Ketamine inhibits endotoxin-induced shock in rats. Anesthesiology 95: 928-932, 2001 .

11. Weigand MA, Horner C, Bardenheuer HJ and Bouchon A: The systemic inflammatory response syndrome. Best Pract Res Clin Anaesthesiol 18: 455-475, 2004.

12. Ware LB and Matthay MA: The acute respiratory distress syndrome. N Engl J Med 342: 1334-1349, 2000.

13. Siler TM, Swierkosz JE, Hyers TM, Fowler AA and Webster RO: Immunoreactive interleukin-1 in bronchoalveolar lavage fluid of high-risk patients and patients with the adult respiratory distress syndrome. Exp Lung Res 15: 881-894, 1989.

14. Aldridge AJ: Role of the neutrophil in septic shock and the adult respiratory distress syndrome. Eur J Surg 168: 204-214, 2002.

15. Molloy RG, Mannick JA and Rodrick ML: Cytokines, sepsis and immunomodulation. Br J Surg 80: 289-297, 1993.

16. Giraud O, Molliex S, Rolland C, Lecon-Malas V, Desmonts JM, Aubier $M$ and Dehoux M: Halogenated anesthetics reduce interleukin-1beta-induced cytokine secretion by rat alveolar type II cells in primary culture. Anesthesiology 98: 74-81, 2003.

17. Zhang G and Ghosh S: Molecular mechanisms of NF-kappaB activation induced by bacterial lipopolysaccharide through Tolllike receptors. J Endotoxin Res 6: 453-457, 2000.

18. De Rossi LW, Brueckmann M, Rex S, Barderschneider M, Buhre W and Rossaint R: Xenon and isoflurane differentially modulate lipopolysaccharide-induced activation of the nuclear transcription factor KB and production of tumor necrosis factoralpha and interleukin-6 in monocytes. Anesth Analg 98: 1007-1012, 2004.

19. Tschaikowsky K, Ritter J, Schroppel K and Kuhn M: Volatile anesthetics differentially affect immunostimulated expression of inducible nitric oxide synthase: role of intracellular calcium. Anesthesiology 92: 1093-1102, 2000.

20. Thomassen MJ, Buhrow LT, Connors MJ, Kaneko FT, Erzurum SC and Kavuru MS: Nitric oxide inhibits inflammatory cytokine production by human alveolar macrophages. Am J Respir Cell Mol Biol 17: 279-283, 1997.

21. Kunz D, Walker G and Pfeilschifter J: Dexamethasone differentially affects interleukin 1B- and cAMP-induced nitric oxide synthase expression in rat renal mesangial cells. Biochem J 304: 3337-3340, 1994. 